\title{
Flow "reflexion" and " refraction" on perforated wall
}

\author{
Václav Tesaŕ ${ }^{*}$ \\ ${ }^{I}$ Institute of Thermomechanics v.v.i., Czech Academy of Sciences, Dolejškova 5, 18200 Praha-Kobylisy, Czech Republic
}

\begin{abstract}
This article presents some results accumulated by author during investigation of an oblique impact of fluid flow on a wall consisting of a spatially periodic rods of very simple oval cross section. The flowfield in the vicinity of the impact is quite complex and strongly Reynolds-number dependent. A part of the jet downstream from the impact is "reflected" from the wall - while the rest, which passes through the empty spaces between the cascade members, leaves the other wall side in what appears to be "refraction" direction.
\end{abstract}

\section{Introduction}

On two different occasions this author was invited to start a research on a fluid flow case that in reality does not exist. In both cases, the proposal was based on the expectation that an inclined impinging jet after hitting a solid wall bounces against it and moves away as presented at left in Fig.1. This flow "reflection" was proposed expected to be similar to a light beam reflected from the surface a mirror or a body moving back from the impact location due to its elasticity. In the case shown in Fig. 1, the "reflected" jet was proposed to propel the a small object - lifting it simultaneously so

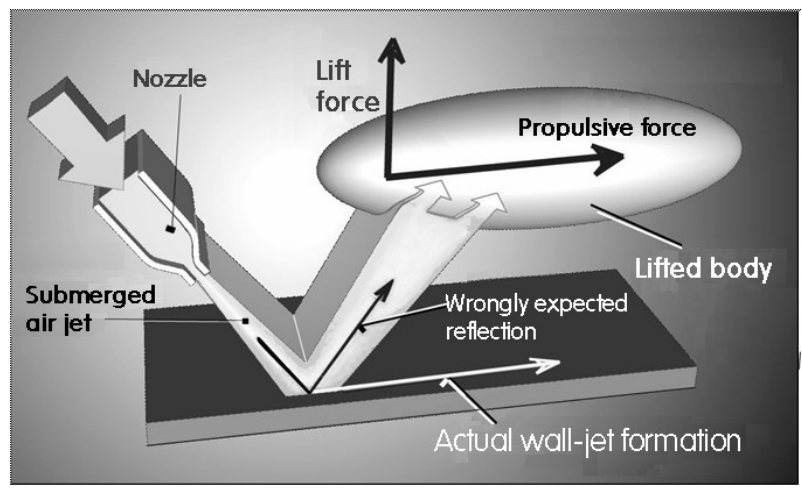

Fig. 1. Wrong idea of transporting by an air jet a small body propelled and lifted by the jet "reflected" from the wall.

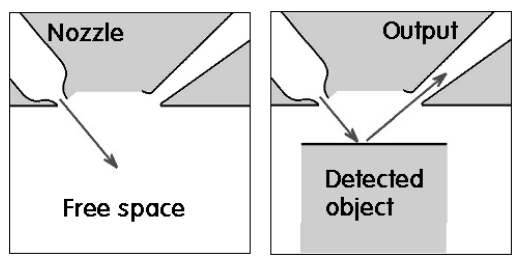

Fig. 2. Essentially the same the same wrong idea of jet behaviour proposed to the author on a different occasion. The "reflected" jet was in this case expected to generate an output signal about the presence of the object. that its motion would not be hindered by friction in the contact with the wall. According to the proposal the nozzles were to be distributed along the body motion trajectory (in Fig. 1 is for simplicity only one of these nozzles). To make the proposal more reliable were then vague and uncertain information that a foreign industrial organisation was rumoured to use a row of such propulsion/lift relay nozzles for moving a weft thread in a textile machine. Indeed, designs of such relay nozzles were later published in [1].

Essentially the same "reflection" of the jet, as was proposed to the author to be used as shown in Fig. 2 in a pneumatic sensor. Its output signal carrying information about presence of a detected object was planned to capture the "reflected" jet.

The proponents of the collaborative investigation of these ideas have even expected that the reflection angle will be equal to the angle of impingement (obviously misguided by the everyday experiences with impacts of elastic bodies).

Of course, what actually happens if a jet impinges obliquely on a solid wall is the generation of the non-

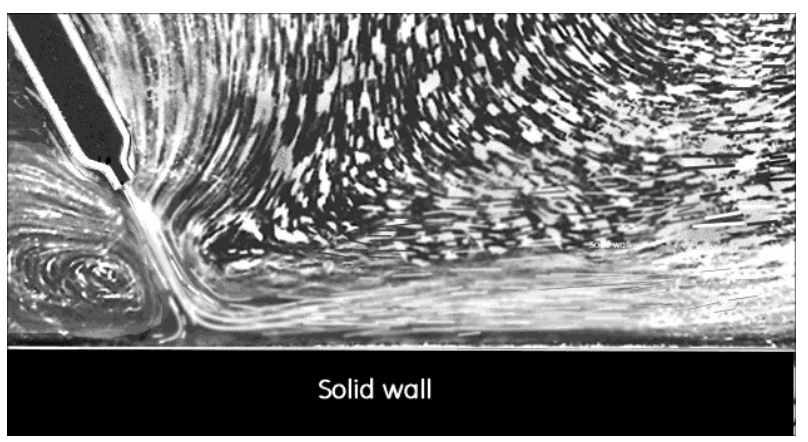

Fig. 3. Two-dimensional jet impact flow visualised by tiny particles in water. The jet issuing from the nozzle at left forms a wall-jet exhibiting no tendency to flow "reflection" or "bouncing". What may look like a small lifting effect at right is wall-jet thickness growth, which cannot generate any useful lift force on tra transported body. 
lifting wall-jet flowing past the wall in contact with it, as shown in the flow-visualisation photograph Fig. 3.

\section{JET "REFLECTION" FROM "LEAKY" WALL}

As a matter of fact, jets may be indeed deflected from their original oblique impact direction in a manner suggesting the "reflection" from the wall $[2,3]$. It is demonstrated in the flow-visualisation photographs Figs. 4 and 7. The trick is to provide the impingement wall with perforations allowing a part of the jet to pass through. Thus apart from the jet impinging flow $X$ (Fig. 5) there is not only the "reflected" flow $Y$ but also the "refracted" flow $Z$ on the opposite side of the wall. The balance between the reflected and refracted parts of the

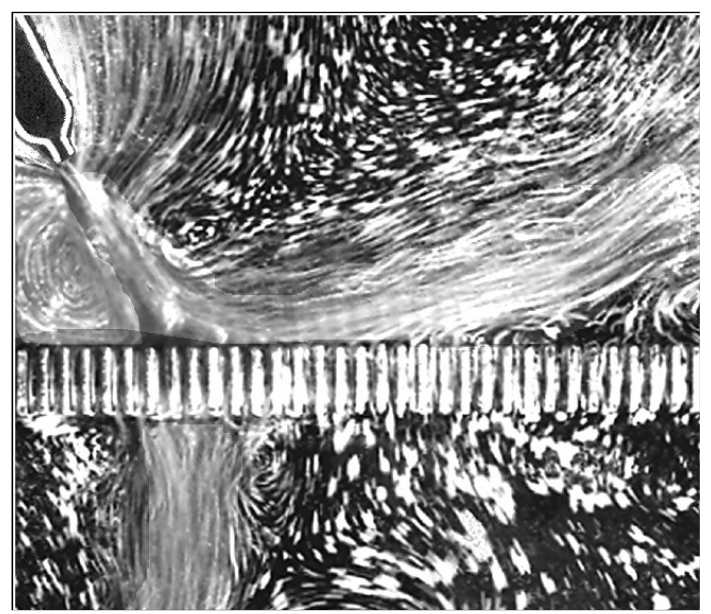

Fig. 4. Visualised oblique impingement of a planar jet on a wall consisting of a large number of thin simple parallel objects with open space between them. The objects are of rectangular cross section with equally rounded leading and trailing edges.

jet may be in principle evaluated by consideration of the vertical components of their moments of momentums in the suitably chosen control volume surrounding the impact. The analysis is, of course, complicated by the far from simple velocity profiles of the flows and also the ) aerodynamic forces acting on the components of the wall. Since the aerodynamic resistance of the wall is

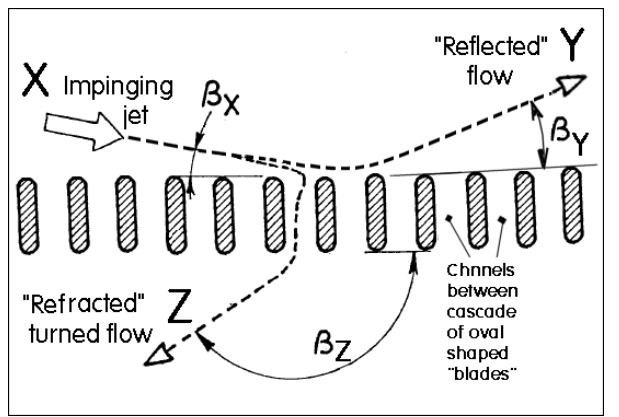

Fig. 5. The splitting of the impinging wall from $X$ into the two outflow components, the reflected $Y$ and refracted $Z$. The angles, especially $\beta z$, vary substantially with varying Reynolds numbers.

not very large (large total area of the perforation openings) some rough estimates are plausible. In principle, seen from the comparison of Fig. 4 (larger impingement angle) and Fig. 7 (larger refracted flow angle), the smaller is the angle $\beta x$, the larger is the refracted flow angle $\beta z$. Important fact which experience has revealed is the angles, especially $\beta z$, varying significantly with the Reynolds number of jet flow.

In author's laboratory investigations, the perforated wall was to be built in a not expensive manner. It was composed from transversally elongated members arranged in parallel. They were all of the same shape, positioned at the same pitch distance $p$ between them Fig. 6. An alternative view of this wall is considering it a cascade of extremely simple airfoils [4]. They were made from round cylinders by flattening between rollers. This was still rather laborious and time consuming so that the whole investigation project was made with the same cascade geometry.

The relative thickness s of the foils was (Fig. 6)

The relative pitch was

$$
\mathrm{s} / l=0.16
$$

$$
\mathrm{p} / \mathrm{l}=0.39
$$

and the relative widths of the open channels between the components

$$
(\mathrm{p}-\mathrm{s}) / l=0.24
$$

Solidity of the perforated wall was

$$
s / p=40 \%
$$

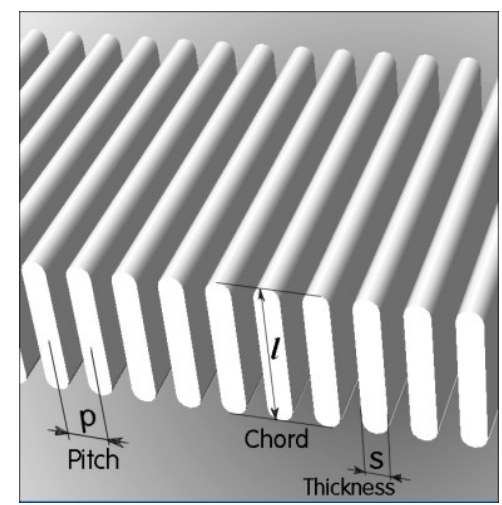

Fig. 6. Author's investigations of the jet reflection and refraction were made with the wall consisting of a cascade of rods made by flattening of what were originally round rods or wires.

\section{THREE APPROACHES TO AUTHOR'S INVESTIGATION}

The strange character of the "reflection" and "refraction" that solved the transport task from Fig. 1 created an interest and even proposals for potential applications of the phenomenoin. Surprisingly, search for previous existing studies in literature did not reveal a slightest trace of any interest paid by earlier investigators. This uniqueness - together with the scientific curiosity about such an interesting but overlooked subject - has led to the decision to investigate these flow configurations in detail. There 
were three approaches, progressing in parallel, the author undertook as follows:

1) Numerical flowfield computations

2) Flow visualisation by particles carried in water

3) Tuft angles measured in airflow photographs

Results of the computations that were run using standard Fluent 6.3.26 software package -- together with the discretisation mesh set up by software Gambit 2.4.6 an example of which is seen in Fig. 9 -- were interesting. It is p-articularly apparent from the several some computation examples of the jet part of the flowfield in Figs. 12,13, 14 and 15. Large series of computation runs were also performed with the flow configuration having the inlet flow through the rectangular cross section channel, Figs. 16 and 17. Unfortunately, especially the latter numerical solutions were not fully comparable with the results obtained with the two other approaches. In fact, the comparisons with flow visualisation in the spaces between the cascade members have revealed in the computed results an absence of several quite important flowfield features. While the visualisations have clearly shown quite important stationary vortical structures influencing especially the inclinations of the exit flowlines, in the computation results these structures were completely absent. The most probable explanation of this mismatch is in the quite small size scale of the investigated flowfields in the channels. The values of the corresponding Reynolds number were mostly in the intervals in which the flows were, while clearly not laminar, not in the regime of fully developed turbulence. The computations in these transitional regimes are usually performed with the turbulence models modified for the low Reynolds numbers conditions. Unfortunately, no modifications were found really reliable. In the here discussed cases they did not met the obvious requirement of smoothly approaching the laminar flow result as the extreme case. The results presented here were computed using the RNG modification approach as was offered in the software package.

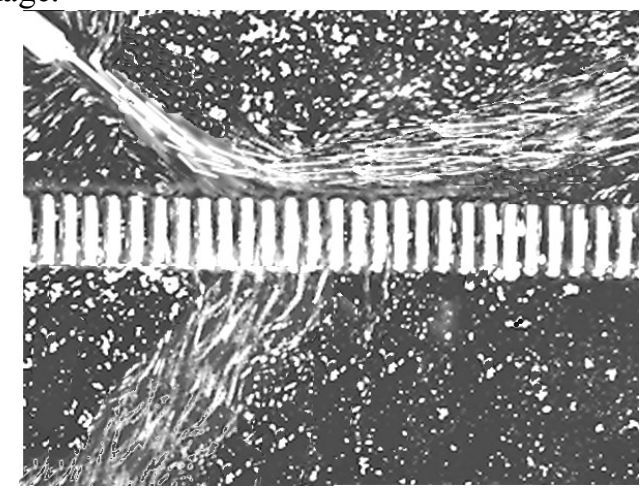

Fig. 7. Another flow visualisation of the obliquely impinging jet. The impinging jet angle $\beta x$ is here smaller than the corresponding one in Fig. 4 - and this has led to the visibly larger is the angle $\beta z$.

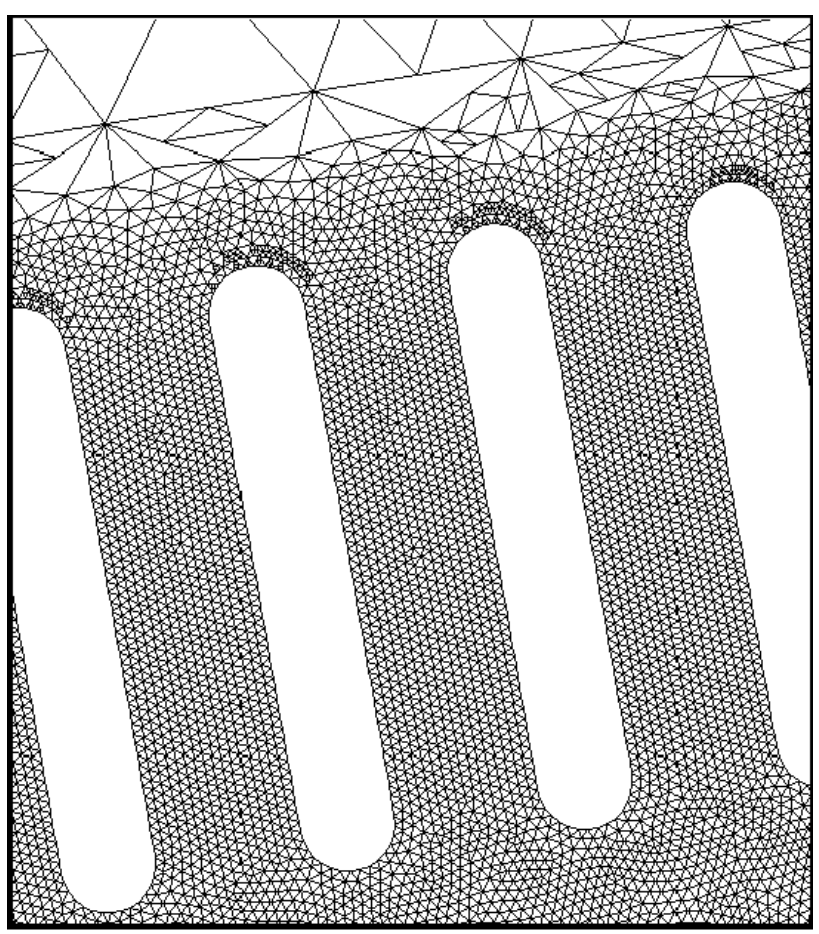

Fig. 8. An example of the discretisation mesh as it was set up in the vicinity of the oval cascade members. The mesh is unstructured triangular, adapted between computation runs by refinement in locations where the absolute magnitudes of local velocity gradient reached values above a limit that was gradually decreased to make the mesh refined. These refinement locations are here seen at the rounded top of the cascade elements, where they improve the solutions inside the boundary layer.

The water flow visualisations were performed in a scaled-up model with water flow seeded with aluminium dust particles. In the photographs these particles were reflecting, dependent of their spatial orientation, some of the illumination light. Typical examples of this visualisation mode are here presented in Figs. 3, 4, and 7. The obtained photographs are very instructive and provide a useful information about the whole flowfield (or about a part of its in the camera field of view). The camera exposures lasted for a time after which the non-stationary moving visualisation particles left in the photographs shorter and longer streakline traces. The effective trace lengths may be decreased by the particle rotation (when in a position with light less reflecting faces) nevertheless as a general rule the lengths of the trajectories in the photographs as, for example, those shown here in Fig. 7 - provide a useful visual information about the local flow directions and even flow velocities. Unfortunately, of course, these visualisation results could be of only qualitative character, not providing numerical data values..

When operated with air, the "refracted" flow leaving the cascade was allowed to exit into the atmosphere. It did so at various outflow angles $\beta z$, (cf. Figs. 5 and 10) which were under some conditions quite surprisingly large. To make the directions of the $Z$ exit flow easily observable, two cascade elements were provided with trailing thin cotton threads, as seen in the 


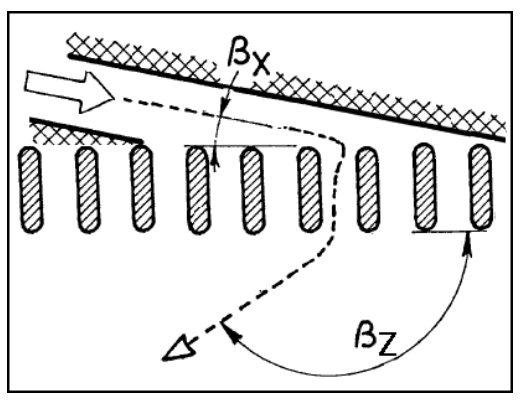

Fig. 9. The configuration of test runs in which, for investigations of the local conditions at the cascade members the jet flows were replaced by well-defined parallel flows in a rectangular channel.
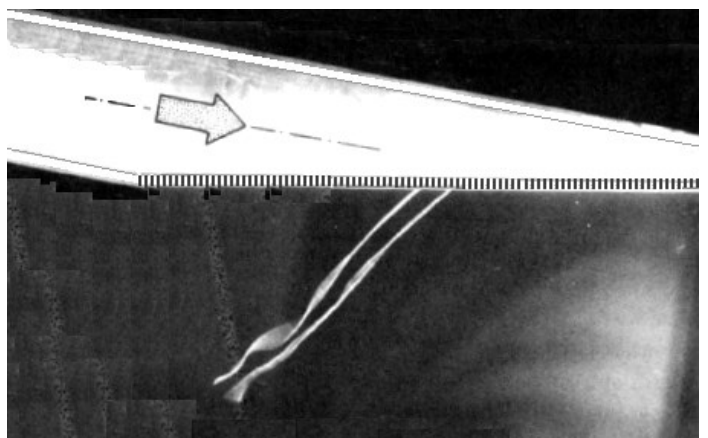

Fig. 10. Typical photograph of the measurements of the refraction angle $\beta_{Z}$. The exit air flow was followed by two cotton threads with their upstream ends fixed to the cascade.

example photograph Fig. 11. In a preliminary series of runs it was ascertained that despite their exposure to the sideways acting gravitational acceleration the threads followed the local air flow lines in the cascade exit with precision better than $1 \%$. It might be noted inFig. 11 that at some higher velocities the threads revealed existence of streamwise oriented vortices.

\section{CHARACTER OF THE COMPUTED JET FLOWS}

Results of the flowfield computations presented here were obtained with the most important one among the geometries of the solution domains - the geometry presented in Fig. 11. The flow in this case was driven by the flow from the nozzle the exit width $b$ of which related to the chord $l$ of cascade elements was

$$
\mathrm{b} / l=0.25
$$

The nozzle was inclined relative to the transversal plane of cascade elements leading edges at an angle 35 deg (Fig. 11). The impact distance from the nozzle exit to this plane was $13.33 \mathrm{~b}$ - a rather large distance for the impact (note that e.g. with the round impinging jets discussed in [10] the distances were only 3.5). The available free channel width between the elements was $0.93 \mathrm{~b}$.

For the lifting_\&_propelling task analogous to what is shown in Fig. 1 there are two important factors: (a) the sufficient height and inclination of the reflected part of the jet above the surface, and (b) no significant loss of jet kinetic energy in the impact.

The first aspect is evaluated for the velocity maxima in Fig. 13. Considering the inevitable loss of kinetic energy in the impact location, the ratio of the angles cannot be of the value 1.0 for the ideal elastic reflection, but the computed value

$$
23.7 / 37.3=0.64
$$

is better that perhaps might be expected. In the next Less optimistic are the computed velocity profiles. An example of them is presented in Fig. 14. The profiles were evaluated along the parallel horizontal planes (or here lines) $a, b$, and $c$ as they are shown in Fig. 11.

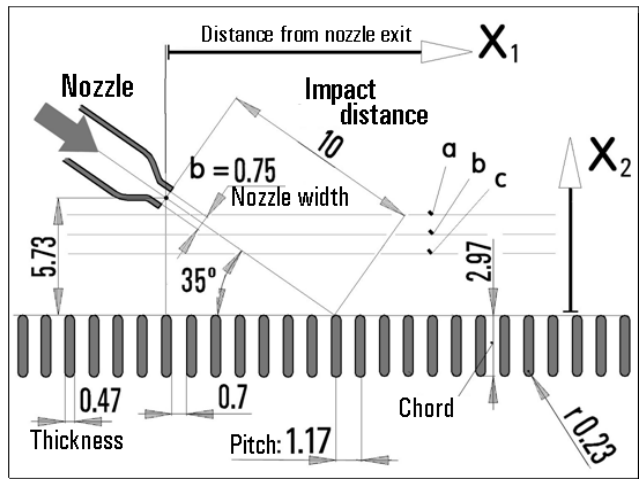

Fig. 11. Geometry of the most important part of the computation domain in the flows driven by the outflow from the inclined nozzle. Note the three parallel lines $a, b$, and c. In them were evaluated the velocity profiles and identified the positions of the jet flow velocity maxima.

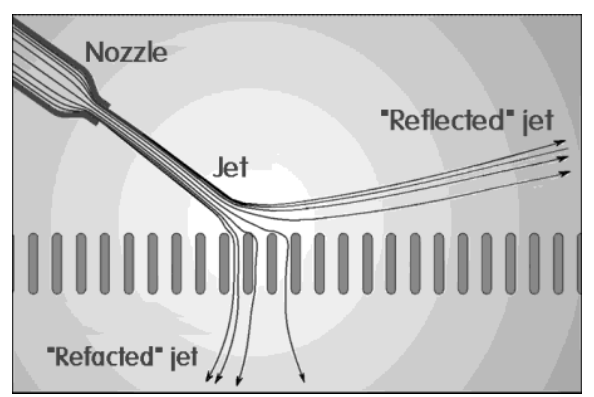

Fig. 12 Typical results of the numerical solution of the flow in the geometry from the previous Fig. 11. Shown in this picture are eight computed pathlines issuing from the nozzle.

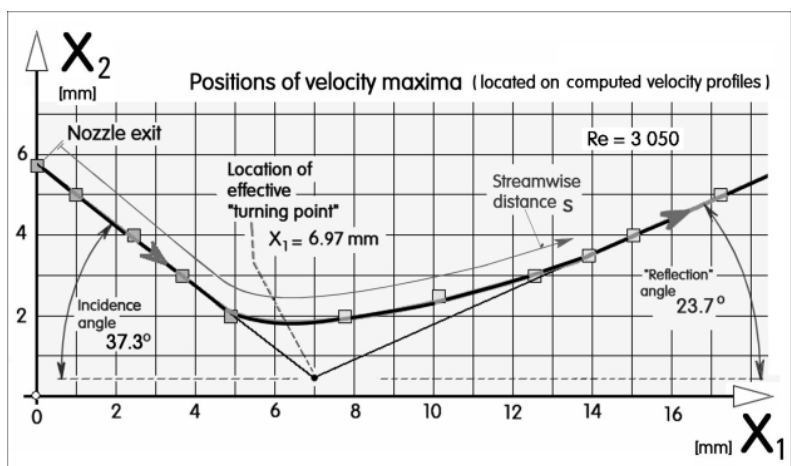

Fig. 13. Mutually connected computed locations of the points in which the jet flow has its local velocity maximum. The angle of reflection at right is smaller than the impingement angle at left, but the difference between them may be considered acceptable. 


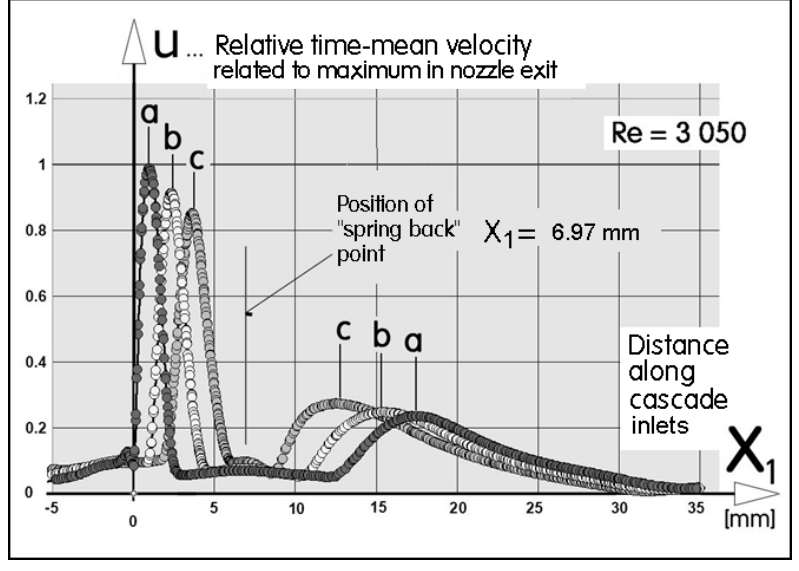

Fig. 14. Typical photograph of the measurements of the refraction angle $\beta_{Z}$. The exit air flow was followed by two cotton threads with their upstream ends fixed to the cascade.

At left are the velocity downstream from the nozzle exit - while at right, with the same labels ( $\mathrm{n}$ inverted order) $\mathrm{c}, \mathrm{b}$, and $\mathrm{a}$ are the following reflected-to-impinging velocity ratios

$\begin{array}{ll}\text { a } & 0.24 \\ \text { b } & 0.27 \\ \text { c } & 0.32\end{array}$

-evidently, the ratio improves with travelled distance. It is the consequence of the rapid decrease on the nozzle side. The conditions would be better with placing the nozzle exit nearer than the present value $13.33 \mathrm{~b}$.

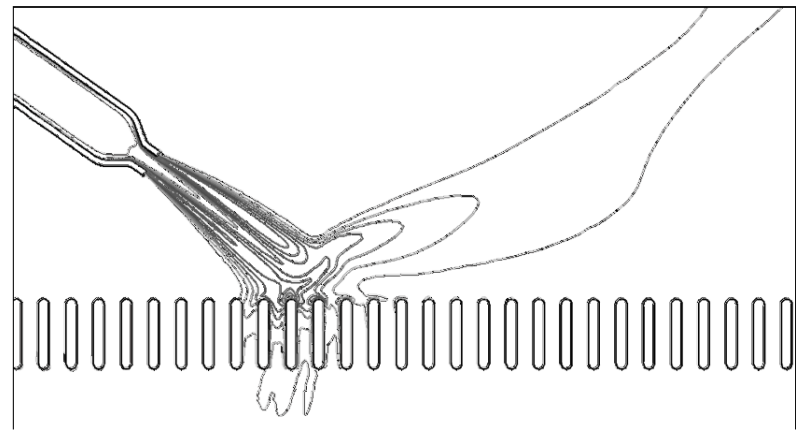

Fig. 15. Computed contours of absolute magnitudes of the air velocity in the same solution domain as in Figs. 12, 13, 14. Remarkable is the complexity of this flowfield.

\section{Flow refraction experiments}

With the complexity typically seen in Fig. 15, it is evident that the details of both reflection and refraction in the investigated flows were difficult to explain. Of course, they have to be a consequence of Reynoldsnumber dependent flow separation and re-attachment effects taking place on the cascade members. The investigation thus concentrated on these details. At this stage were necessary some simplifications. With the flowfield driven by the nozzle efflux, different cascade members had different working conditions and evaluation of a universally valid conclusions for them was out of question. As was already mentioned in association with the Figs. 9 and 10, to have the easier conditions for investigating details of flow past the cascade elements, it was decided to re-arrange the test configuration to so that most (apart from few ones at the cascade ends) the foils obtained everywhere the same inlet angle $\beta x$. This was achieved by bringing to the interaction cavity the inlet flow not from the nozzle but through a parallel-wall channel (of rectangular cross section). For the laboratory tests were made available four such inclined inlet channels, with the inflow angles $\beta x=30^{\circ}, 20^{\circ}, 13^{\circ}$, and $10^{\circ}$. Such configuration was also set up for numerical computations. The solution domain was as in Fig. 16.

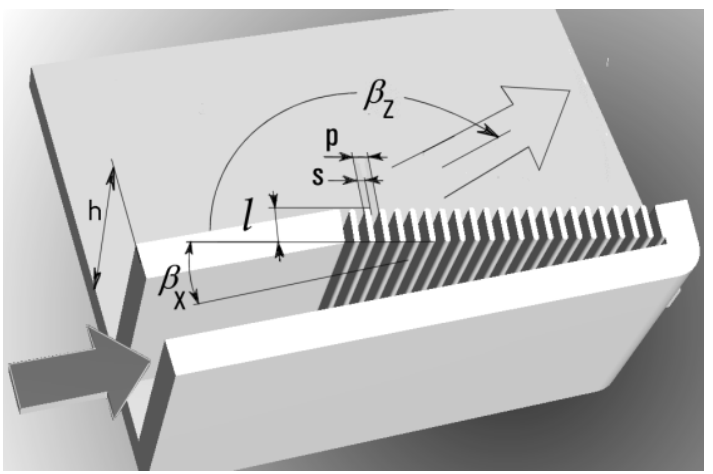

Fig. 16. Geometry of the computation domain with the inlet channel of rectangular cross section. and absence of the reflected flow. This different character of the domain was introduced to make possible a better detailed study of the flow past the cascade elements.

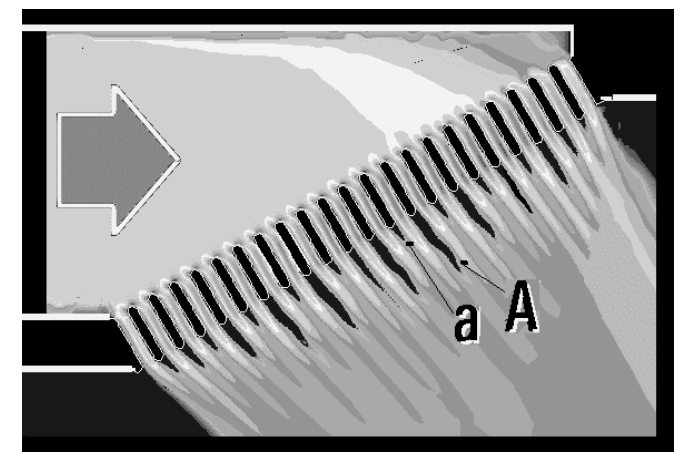

Fig. 17. Computed distribution of specific kinetic energy of flow in the domain of Fig. 16. In contrast to the flowfield driven by the jet (Fig. 11) the main part of the cascade members are exposed to the same inlet conditions. Surprisingly, the cascade elements form pairs, here apparent in the size of the element wakes $A$ and $a$.

The most surprising effect observed in the results of these computations is seen in Fig. 17. Instead of the (naturally expected) identity of all flows past the cascade members - which were identical - the flows tend to appear in pairs with differently behaving pair elements. In Fig. 17 it is evident in the alternating size of the wake downstream from the cascade. Between two elements with the longer wake $A$ there are (geometrically the same) elements with the shorter wake a. 


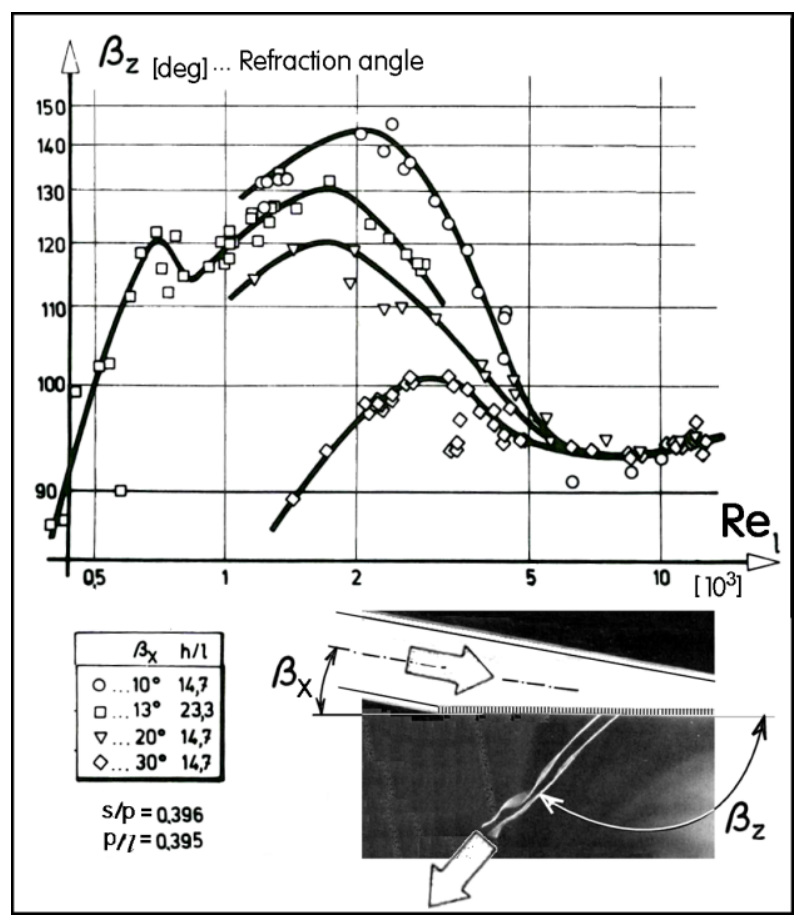

Fig. 18. Experimental refraction angle results. The angles $\beta z$ are strongly dependent on Reynolds number $\operatorname{Re}_{l}$ evaluated from the cascade chord length $l$.

\section{Flow details inside the cascade}

Presented in Fig. 18 are the values of refraction angle $\beta z$ obtained by measurements of inclinations of threads in the exit air flow, the method presented in association with Figs. 9 and 10. On the horizontal co-ordinate in Fig. 18 are values of Reynolds number $\mathrm{Re}_{l}$ evaluated from the measured air flow velocity and chord length $l$ of the cascade members. There are four data groups, each for the constant impact angle $\beta x$. determined by the inclination of the flow in the inflow channel.

Immediately apparent in this diagram Fig. 18 is the strong dependence of the behaviour on the flow rate magnitude. The effects are the more pronounced the smaller is the inflow angle $\beta x$. The largest angular variations were found for the smallest $\beta x=10^{\circ}$. The other, not expected fact seen in Fig. 18 is the large magnitude of the refraction angles $\beta z$. Note that all data values there are larger than the angle $\beta z=90$ deg that might be expected as the direction imparted by the relatively long and straight channel between the cascade members. Of course, with the blunt round trailing edges of the cascade elements, the angle $\beta z$ may be changed easily. Also, the 90 deg angle would require the channel between elements to be fully filled by a parallel flow. The flow visualisation in Fig. 19 shows that the flow actually uses only a small part of the available channel cross section. This rather thin flowpath may be easily directed to aim elsewhere by an action at its end (at the elements' trailing edges). The rest of the cross section in the channel - not used by the flowpath - is occupied by stationary vortices. In the photograph Fig. 19 are seen only the vortices $A$. They are formed by the flow separation from the wall stagnation points $S$ at the leading edges, shown in Fig. 20. According to

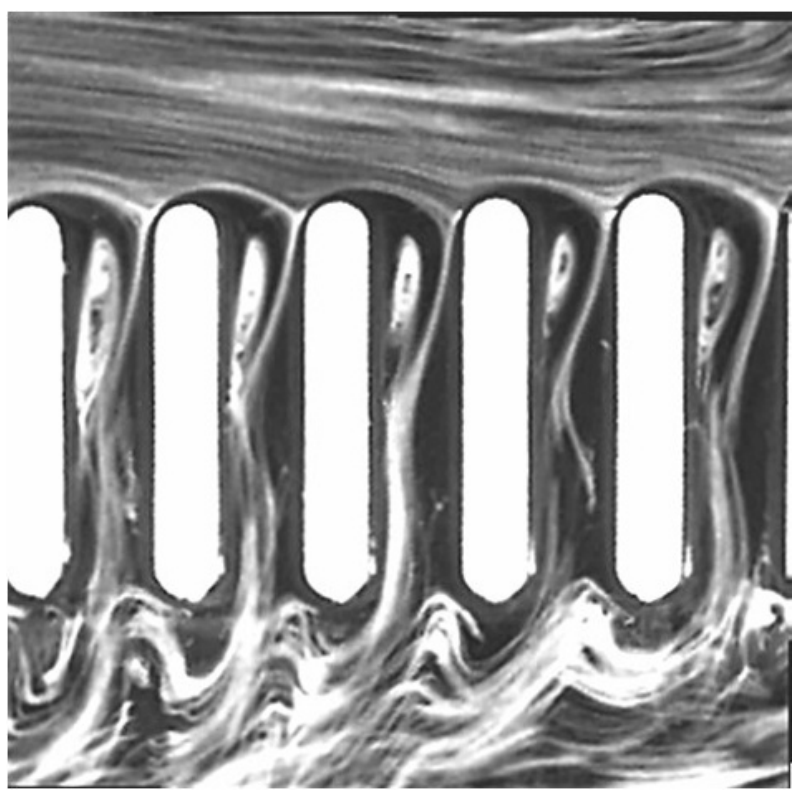

Fig. 19. Typical photograph of small detail of visualised blade cascade in water seeded by aluminium dust particles. Most important role play the stationary vortices, indicated in Fig. 20.

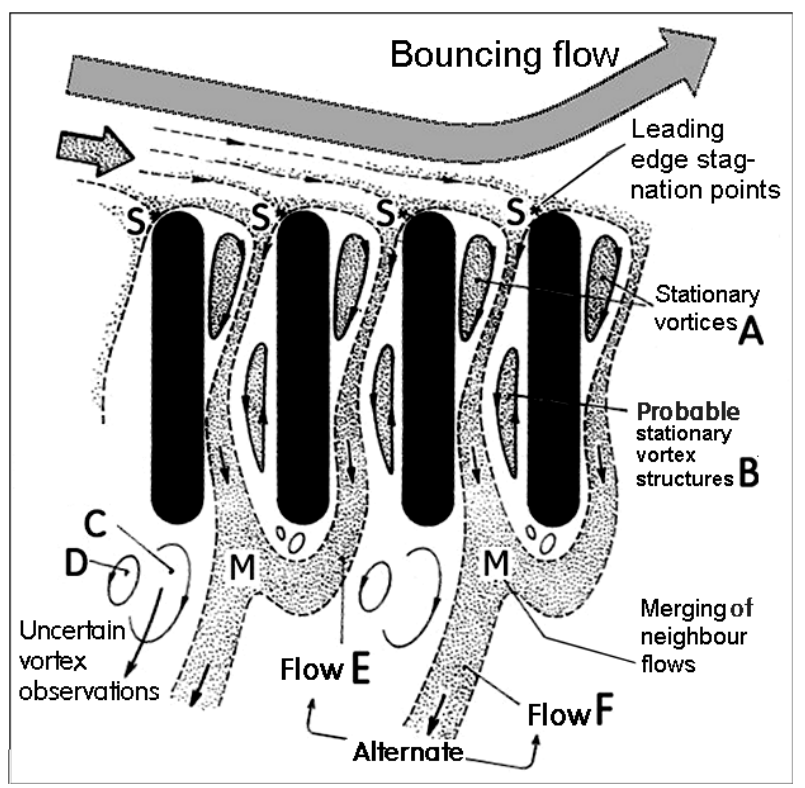

Fig. 20. Objects and flow configurations seen in photographs like the one in Fig. 19. Presence of the vortices B (in many flow visualisations not seen (cf. Fig. 19 above) were inferred from other aspects of the flow. Quite strange effect is the merging of a pair of neighbour flows at the blade's trailing edge.

Helmholtz' [11] vortex conservation law (it is impossible an inviscid fluid to produce or destroy a single vortex - there must be two contra-rotating vortices) there should be inside the channel another system of vortices of opposite sense of rotation. These inferred vortices were marked B as indicated in Fig. 20. The flow visualisation technique originally used by the author initially failed to show them - they are invisible 


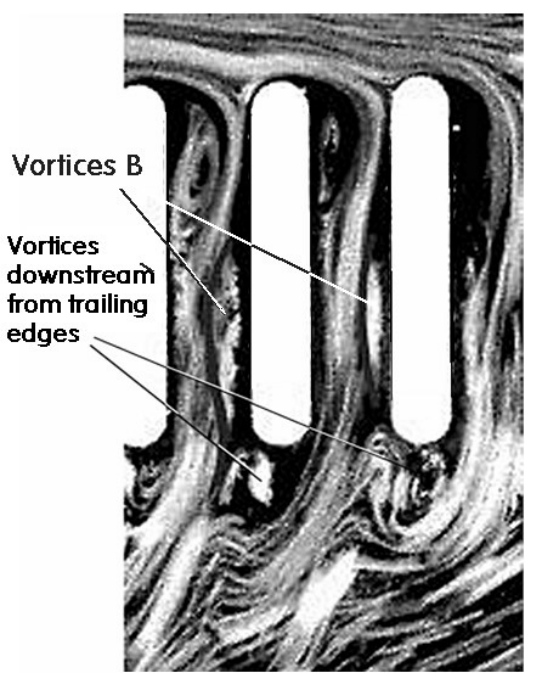

Fig. 21. Typical photograph of the measurements of the refraction angle $\beta z$. This is the case that finally demonstrated the existence of the vortices B. On the other hand, it revealed also the presence of vortices $C$ and D (Fig. 20) at the trailing edges of the blades.

in Fig. 19 (absence of the seeded particles crossing their border line). Their later appearance in Fig. 21 was achieved with the slightly modified visualisation approach.

Important for the demonstrated exit flow refraction are the vortical motions located downstream from the end of the blades. These motions are in Fig. 20 labelled $C$ and $D-$ and clearly recognisable in Fig. 21. In many flow visualisation photographs their clear observation was nor possible or complicated due to the significant unsteadiness of the flow in these locations.

Unexpected and indeed quite unique as unusual fluid mechanical phenomenon is the pairing flow effect. It is obviously associated with the pairing of wakes mentioned above in association with Fig. 17. Under some conditions, the wakes of neighbouring cascade members exhibited merging into only one wake. This merging is marked as $\mathrm{M}$ in the explanatory drawing Fig. 20. There are also conditions under which the flows $E$ and F (indicated Fig. 20) alternate between the two merging regimes (left and right). It is obvious that in these flows there is a wealth of so far unknown or little known phenomena.

\section{Potential applications}

Due to very limited financial means available for this particular research, it was not possible to perform investigations of the described phenomena in practical engineering uses - despite the fact that it is possible to name quite a number of potential uses. It was. here decided to mention briefly at least four of cases - in order to indicate why a continuation research might be worth the time and effort.

In the first case, the horizontal cascade and an air jet blowing from an obliquely directed nozzle may be indeed used for moving threads and yarns in textile

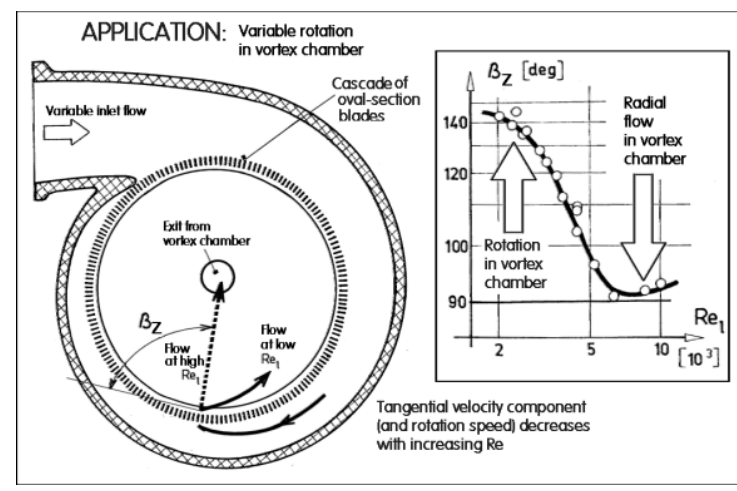

Fig. 22. An application of the investigated variations of the exit angle. The fluid enters the vortex chamber past the cascade and its varying flow direction may be arranged to keep a constant pressure difference across the device in spite of varying flow rate.

machines, in particular pneumatic looms [12]. In particular, a pair of nozzles above the loom reed was demonstrated [5] to be able to drive by air jets so that the weft could be controllably inserted into the shed formed of warp threads.

In another application, the flow was arranged in a manner essentially similar to Fig. 16 but with the flow delivering channel scrolled into a volute - as shown in Fig. 22. The small cascade members are oriented radially, pointing inwards into the vortex chamber (with the central exit) which is placed in the centre. The automatic change of the inclination $\beta z$ in this case secures a constant pressure drop (- the result is a simple no-moving-part pressure controller. At a large flow rate is in the vortex chamber almost no rotation. Thus the pressure drop across the whole device is not large. Gradually decreasing flow rate causes an increase of the angle $\beta z$ so that the fluid inside the chamber rotates increasingly fast. The aerodynamic resistance increases due to the centrifugal forces. This way the pressure drop across the device remains constant over a large range of flows.

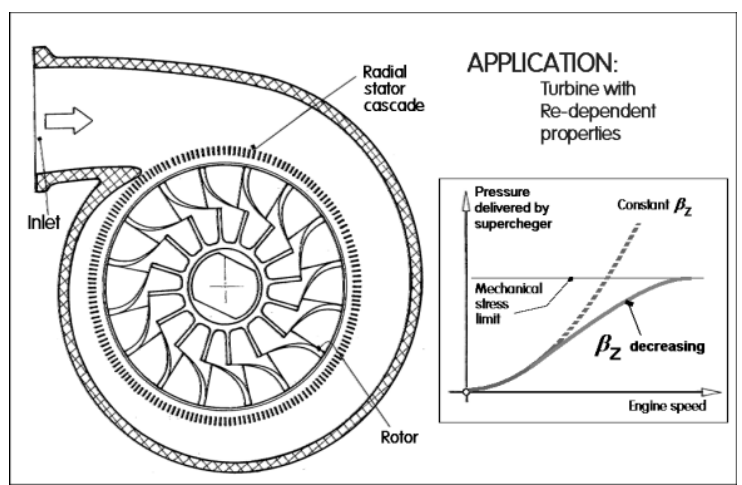

Fig. 23 Another application in a radial turbine. The decreasing inclination $\beta z$ of the stator exit flow prevents surpassing the pressure limit above which would be endangered engine life.

There is a number of potential uses in small and inexpensive turbines, according to Fig. 23 - where the stator is for simplicity of explanation the same as in Fig. 22. This stator adapts its operation in a much simpler way than with the usual system of mechanically adjustable stator blades. 
One of the examples is a small water turbine driving a synchronous AC electricity generator $[7,8]$. The basic requirement is keeping a constant rotation speed - and hence constant AC current frequency. In an uncontrolled turbine, decreasing the supplied water flow rate would decrease the speed and hence unacceptably decrease the frequency. According to Fig. 22, the decreasing flow rate would increase the inclination angle $\beta_{z}$ and thus the resultant rotation speed - so that the frequency remains constant.

The same turbine stator principle may be used in a small and inexpensive combustion-engine supercharger, driven by exhaust gas flow [7, 9]. An increase in engine speed in the usual turbines causes an increase in the inlet pressure delivered to the engine - to a level in which the pressure would reach a magnitude not acceptable from the engine life point of view. According to Fig. 23, the pressure will remain below the dangerous limit because the Reynolds-number dependent "refraction" will cause a decrease of the $\beta z$ stator exit angles and hence the turbine rotor speed.

\section{Conclusions}

Purpose of this paper is to create an interest in the so far practically unknown case of fluid mechanics - the configuration with a cascade of simply oval-shaped blades. Impinging upon it, the flow changes its direction. This it can generate the otherwise "impossible" flow reflection - and Reynolds-number dependent refraction. Discussed are two studied alternatives: one with an obliquely impinging jet generated in a nozzle - and the other, with the inflow guided in a parallel walls channels. Briefly mentioned are also four practical engineering applications.

Author has received research grant Nr. 17-08218S from GAČR, the Czech Republic Grant Agency. He has also obtained institutional support RVO: 61388998.

\section{References}

1. P. Gunneman, Düse für eine pneumatische Webmaschie. European Patent Application 0423066 A1, filed Sept. 1990

2. V. Tesař, P. Smolka, „Exit angles of air flow passing through obliquely positioned perforeted wall', (in Czech), Proceedings of Conf. 'Aplikácia experimentálnych metód v mechanike tekutín', Tatranske Matliare, Slovakia, April 1989

3. V. Tesař , "Reflection" of a fluid jet from a (perforated) wall: a flow visualisation study, Proceedings of the 5th International Symposium Flow Visualisation V, Prague Czech Rep., p. 640, 1989

4. N. Scholz, Aerodynamik der Schauffelgitter, G. Barun Verlag, 1965

5. V. Tesař, Aerodynamic investigations of weaving reed, Proceedings of CTU seminar 94, p. 167, Prague Czech Rep., Jan. 1994

6. V. Tesar., Reynolds-number dependent flow turning by a fixed cascade, Proc. of XI. Internat. Conference Recirkulacja, Bydgoszcz, Poland, p. 265, 2001

7. V. Tesar̆, Turbine varying with its properties in dependence on fluid flow rate, Czech Rep. Patent Application PV6901-90 filed 29th.Dec.1990

8. P. C. Sen, Principles of Electric Machines and Power Electronics, 2nd ed., John Wiley \& Sons, 1997

9. W.W. Pulkrabek, Engineering Fundamentals of the Internal Combustion Engine. Prentice Hall 1997

10. V. Tesař, EPJ Web of Conferences, 45, 01129, 2013

11. H. Helmholtz, Uber Integrale der hydrodynamischen Gleichungen, welche den Wirbelbewegungen entsprechen, Crelles Journal fiir die reine und angewandte Mathematik, $55,25,1858$

12. V. Tesař, Development on weft insertion nozzles for pneumatic shuttle-less looms at CVUT in 1980‘s, Proc. of 14 Scientific-pedagogical Conf., p. 165, Harrachov, Czech Republic, 1995 